\title{
Driver Assistance System Based on Video Image Processing for Emergency Case in Tunnel
}

\author{
Huthaifa Ahmad Al_Issa \\ Department of Electrical and Electronics Engineering, Faculty of Engineering, Al-Balqa Applied University, Irbid Al-Huson, Jordan
}

\section{Email address:}

alissahu@yahoo.com

\section{To cite this article:}

Huthaifa Ahmad Al_Issa. Driver Assistance System Based on Video Image Processing for Emergency Case in Tunnel. American Journal of Networks and Communications. Vol. 4, No. 1, 2015, pp. 5-9. doi: 10.11648/j.ajnc.20150401.12

\begin{abstract}
This paper proposes architecture for detecting accidents system based on image processing techniques for emergency case in tunnel, as well as the technical challenges that had to be overcome to ensure that technology successfully operated under all conditions. The advantages of this method include such benefits as Non-use of sensors, low cost and easy setup and relatively good accuracy and speed. Because this method has been implemented using image processing and MATLAB software, production costs are low while achieving high speed and accuracy. Method presented in this research is simple and there is no need to use sensors that have been commonly used to detect traffic in the past. This research can be enhanced by helping out the driver assistance system, this is accomplished by informing the public traffic about accidents in specific areas so that they can avoid those routes.
\end{abstract}

Keywords: Driver Assistance System, Video Image Processing, Traffic Safety

\section{Introduction}

Video traffic detection is one of the most important applications for the combination of the two important toolboxes in MATLAB which are video processing toolbox and image processing toolbox. The main idea of the traffic detection application is to keep the controller aware of any new or emergency case in the traffic such as: car crash, water flood or any other unwanted case that would affect the traffic negatively. Once the emergency case was noticed and the alarm was triggered, the controller could handle that specific case as soon as possible $[1,2]$.

The image processing toolbox can help by some applications for image processing, analysis, visualization and algorithm development. These applications are provided as an extensive set of reference-standard algorithms and functions. Meanwhile, the video processing toolbox may help in analyzing the video and developing algorithms to read or detect specific parts in the video, such as: dark areas, light areas, borders and so on.

The video processing toolbox application can be used in both cases of video: offline and online videos, in the case of the offline video: the video is stored in the path of the MATLAB in the pc, where the user can upload the video to the program using a specific command to read and analyze the requested data. While in the case of the online video, the video will be broadcasted from a live video, similar to the procedure of this research, where the video should be coming from a live camera that is spotted somewhere in a tunnel to monitor the case of the traffic in order to be analyzed and decided whether the case is normal or not $[3,4]$.

The video traffic detection function has the following advantages: Allows analyzing the traffic, helps in handling the emergency cases in a short time, can be improved to detect the faces which may help to catch or track the criminals, moreover, surely it can help in traffic management.

\section{Digital Image Processing}

An image may be defined as a two-dimensional function $f$ $=(x, y)$, where $x$ and $y$ are spatial (plane) coordinates, the amplitude of $f$ at any pair of coordinates $(x, y)$ is called the intensity or gray level of the image at that point. When $x, y$ and the amplitude values of $f$ are all finite, discrete quantities, we can call the image is as a digital image. The field of digital image processing refers to processing digital images by means of a digital computer. Note that the digital image is composed of a finite number of elements, each of which has a particular location and value. These elements are referred to as picture elements, image elements, and pixels. An image may be considered to contain sub-images sometimes referred 
to as regions-of-interest, and transformations [5].

Images based on radiation from the EM spectrum are the most familiar, especially images in the X-ray and visual bands of the spectrum. Electromagnetic waves can be conceptualized as propagating sinusoidal waves of varying wavelengths, or they can be thought of as a stream of mass less particles, each traveling in a wavelike pattern and moving at the speed of light. Each mass less particle contains a certain amount (or bundle) of energy. Each bundle of energy is called a photon. If spectral bands are grouped according to energy per photon, we obtain the spectrum as shown in figure 1, ranging from gamma rays (highest energy) at one end to radio waves (lowest energy) at the other. The bands are shown shaded to convey the fact that bands of the EM spectrum are not distinct but rather transition smoothly from one to the other [6].

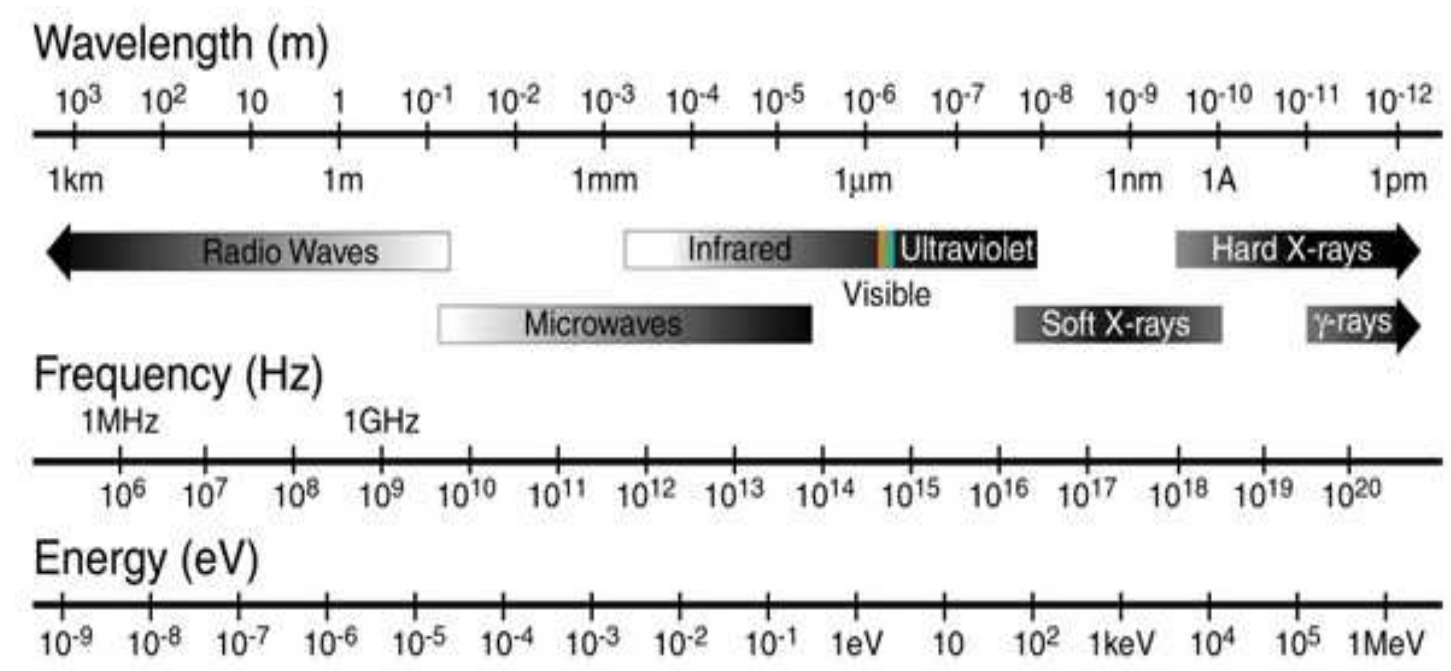

Figure 1. The electromagnetic spectrum arranged according to energy per photon.

\section{Discussion of the Theory}

Nowadays, tunnels handle large volumes of automobile traffic, sometimes moving at expressway speeds. In the narrow, confined environment of a tunnel, even minor incidents can cause deadly disasters, since critical, catastrophic incidents may occur even faster than on surface highways. An important objective of traffic management will be to identify potential sources of danger even before they reach the tunnel. In the face of the additional risks presented by subterranean accidents, every tunnel operator would like to be alerted early of any hazardous conditions. To pave the way to realizing this ideal, and the objective of this research is the traffic control in tunnel to ensuring the safety of the road users, and maximizing the availability of these structures [7].

All systems which are managed by traffic controller with its software that is capable of executing different logical, sequence, time, numeric arithmetic, regulative and communication functions. All subsystems, such as traffic lights, LED signalization, traffic count with inductive loops, emergency call system, video control, fire alarm, management of low voltage equipment and power supply, are connected to regional control center, where computers and supervisors are monitoring tunnels 24/7. Traffic safety is the key word. Function of Automatic Incident Detection (AID) is normally based on computer-based analysis of video image streams generated from cameras set up to view tunnel traffic [8].
Due to Needs a huge number of staff required to monitor each traffic area, and to the difficulties to describe the emergency case clearly to the operation center to handle the problem quickly, the need for automated detect and alarm system is essential. This research presents a method for estimating car accidents in the traffic using Image, this is done by using the camera images captured from the highway and videos taken are converted to the image sequences. The proposed algorithm expected to help in the traffic management field by applying the video processing functions in the traffic field, help in detecting the car crash cases in the tunnel, and help in detecting the water flood cases in the tunnel.

\section{Methodology}

In this research, we used the MATLAB software, we used a combination of two main toolboxes in MATLAB, video processing toolbox and image processing toolbox.

Image processing toolbox provides a comprehensive set of reference-standard algorithms, functions, and applications for image processing, analysis, visualization, and algorithm development. We can perform image enhancement, feature detection, noise reduction, image segmentation, geometric transformations, and image registration. Image processing toolbox supports a diverse set of image types, including high dynamic range, gig pixel resolution $[9,10]$.

Video processing toolbox is one of the main toolboxes in the image field in MATLAB, this toolbox offers the user 
many functions in order to analyze the videos and get the required data from those videos [11]. This type of processing is critical in systems that have live video or where the video data is so large that loading the entire set into the workspaces is inefficient. Computer vision system toolbox supports a stream processing architecture through system objects for use in MATLAB and blocks for use in Simulink.

The code was run in MATLAB it due some analysis in data from a film, the analysis includes finding number of frames, number of background frames, and due some process on dust, dirt, and brightness (based on night time, pixel segment with spatial temporal techniques) according to normal circumstances data, and focus on spots experienced notable changes. The code set a threshold on how much size a car can be recognized.

We set a threshold for the changes which are based on parameters, in order to reduce false alarms. Once an accident is recorded a peep alarm runs for three times every half a second. One drawback of the experiment that in order to monitor all the accidents a three seconds interval have to be for every two concessive accidents.

Video processing toolbox has many functions that help in analyzing the videos like:

- Video Reader:
Video reader is a function that is used to read the video data from a file into MATLAB workspace (in this research the file is a live cam).

- Implay:

The function IMPLAY is a function that opens a movie player for showing MATLAB image sequence (or image stacks), showing videos, and movies.

- Image Comparison: Imshowpair:

As this research requires a comparison between images, we need have a function to compare the images in order to analyze it. The function IMSHOWPAIR is a very good function to compare the differences between two different images.

- Alarming:

In this research we used alarming which is called beep, where beep is a sound that can be produced in MATLAB, so we used as alarm due to many reasons, one of them is our research needs to get a high speed response so the beep sound will keep running until the controller notices it and make a fast response. Another reason for choosing beep, as an alarm that its shorter to be generated and more effective tool to use in such cases.

The flow chart for this algorithm is describing below in figure 2 .

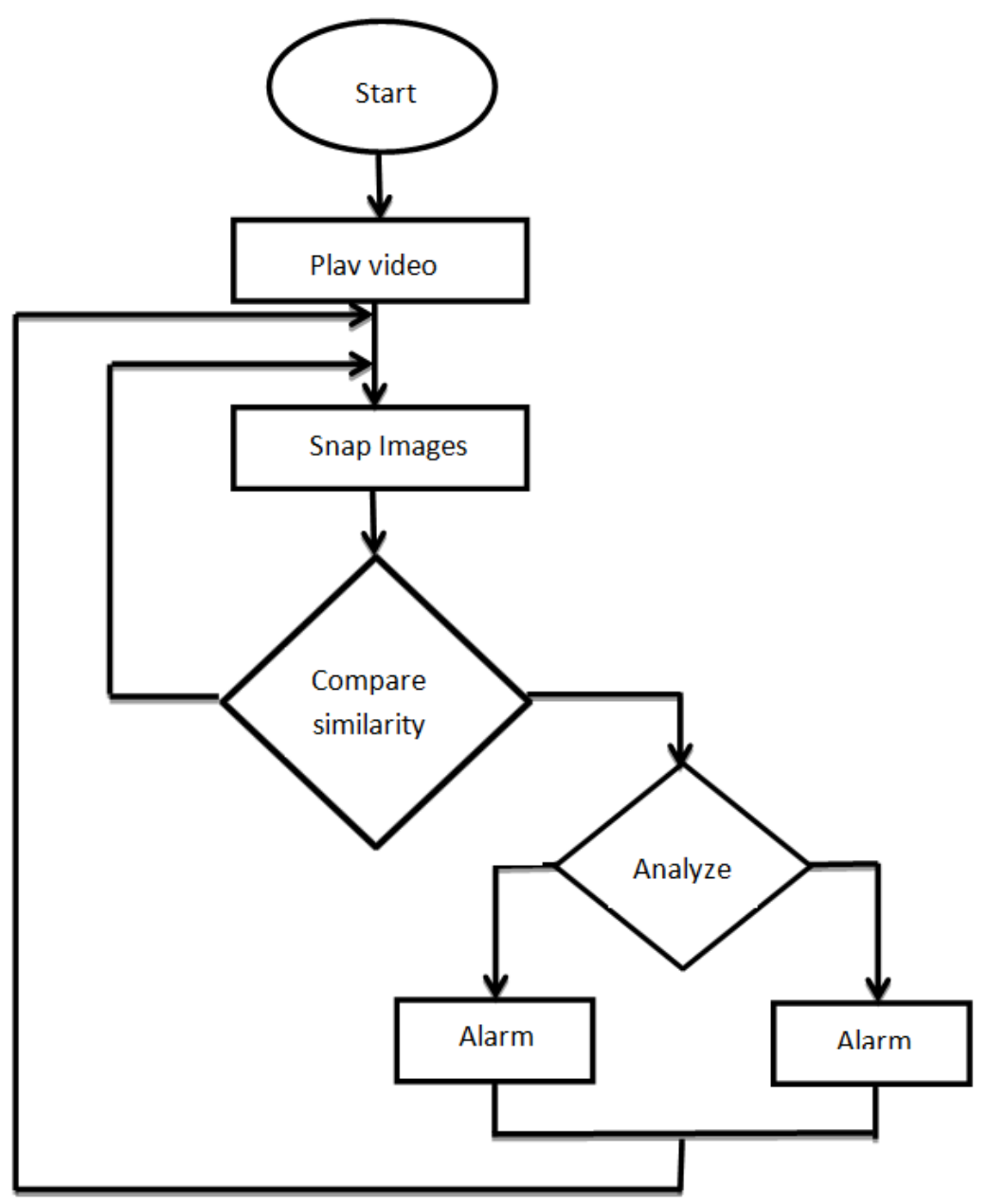

Figure 2. Flow chart describes the algorithm for this research. 


\section{Research Results}

This program ensures the movement of cars and detects if accidents have occurred by seeking cars which are placed horizontally or the accumulation of dust and dirt. The program works in 4 second intervals in which the program checks for accidents by the above mentioned method. If there is more than one accident in each 4 second block, the number of accidents is shown above the second illustration, as shown in figure 3.

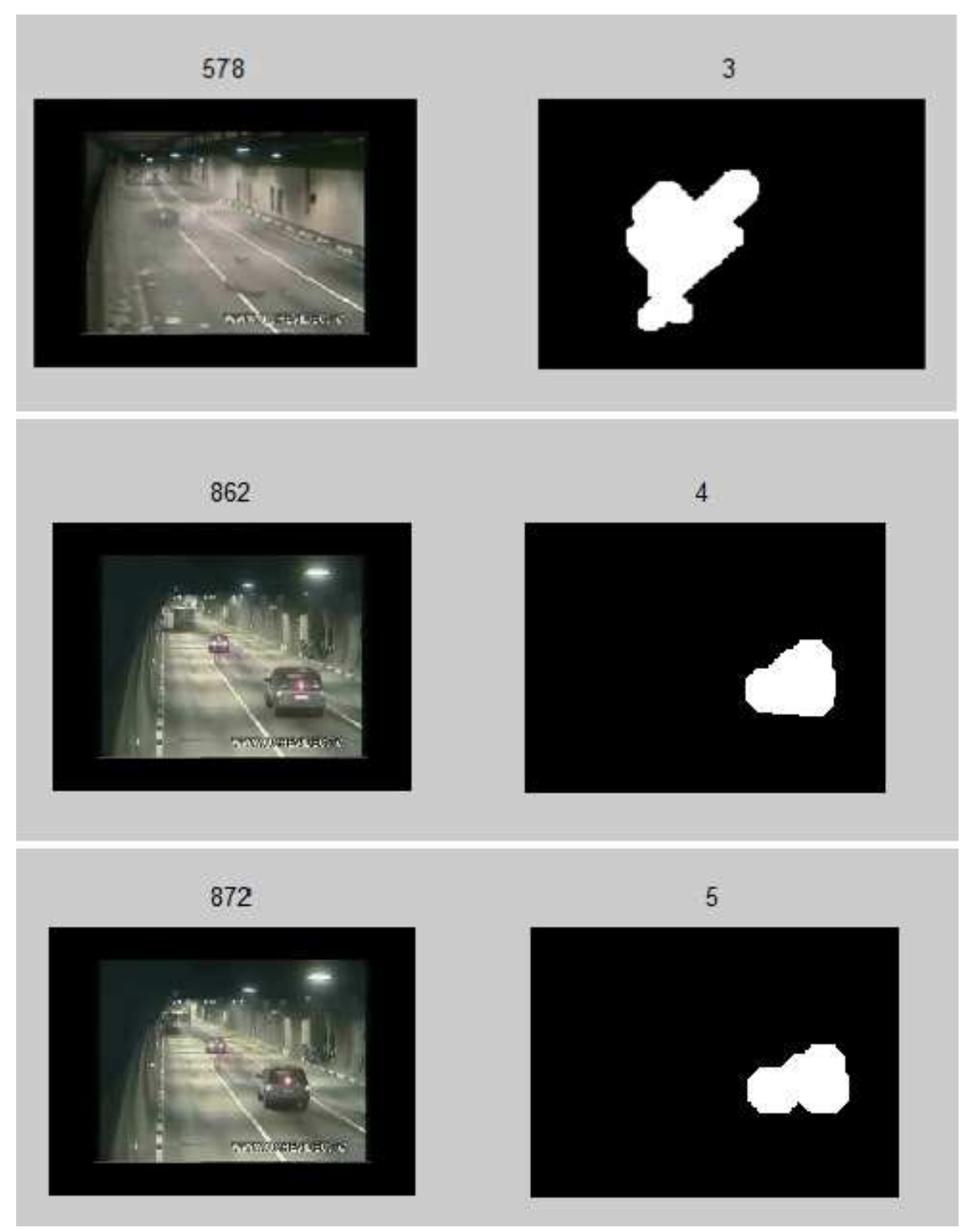

Figure 3. The code's generated images for a frame's accident recorded inside the tunnel.

This research can be enhanced by helping out the driver assistance system, this is accomplished by informing the public traffic about accidents in specific areas so that they can avoid those routes.

\section{Conclusion}

This paper presents a method for estimating car accidents in the traffic using image, this is done by using the camera images captured from the highway and videos taken are converted to the image sequences. Each image is processed separately and the advantages of this method include such benefits as Non-use of sensors, low cost and easy setup and relatively good accuracy and speed. Because this method has been implemented using image processing and MATLAB software, production costs are low while achieving high speed and accuracy. Method presented in this research is simple and there is no need to use sensors that have been commonly used to detect traffic in the past. One of the disadvantages of this method is extremely sincerity to the light this problem can be overcome by using specific camera filters during imaging processing or change in MATLAB code, this method can be used to detect rood accident. 


\section{References}

[1] HunjaeYoo; Ukil Yang; KwanghoonSohn "GradientEnhancing Conversion for Illumination-Robust Lane Detection", Intelligent Transportation Systems, IEEE Transactions on, On page(s): 1083 - 1094 Volume: 14, Issue: 3, Sept. 2013

[2] Farrell, J.A. Givargis, T.D. Barth, M.J. "Realtime differential carrier phase GPS-aided INS".IEEE Transactions on Control Systems Technology,2000,8 (4):709-720.

[3] A. López, C. Caṇero, J. Serrat, J. Saludes, F. Lumbreras, and T. Graf, "Detection of Lane Markings based on Ridgeness and RANSAC," IEEE Conference on Intelligent Transportation Systems, 2005, pp. 733-738.

[4] B. F. Wu, C. J. Chen, Y. P. Hsu, and M. W. Chung, "A DSPbased lane departure warning system," in Mathematical Methods and Computational Techniques in Electrical Engineering, Proc. 8th WSEASInt. Conf. Bucharest, 2006, pp. 240-245

[5] M. J. Jeng, P. C. Hsueh, C. W. Yeh, P. Y. Hsiao, C. H. Cheng, and L. B. Chang, "Real time mobile lane detection system on PXA255 embedded system," International Journal of Circuits, Systems and Signal Processing, vol. 1, no. 2, pp. 177-181, 2007.

http://www.naun.org/multimedia/NAUN/circuitssystemssignal /cssp-31.pdf

[6] T. Al Smadi, Computing Simulation for Traffic Control over Two Intersections, Journal of Advanced Computer Science and Technology Research, Vol 1, No 1.pp. 1024,2011.http://www.sign-ificance.co.uk/dsr/index.php/JACSTR/article/view/38

[7] Chen, L.; Li, Q.; Li, M.; Zhang, L.; Mao, Q. Design of a Multi-Sensor Cooperation Travel Environment Perception System for Autonomous Vehicle. Sensors 2012,12, 1238612404. http://www.mdpi.com/1424-8220/12/9/12386

[8] Wang Y., Teoh E.K, Shen D. "Lane detection using Bsnake".Int. Conf. Information Intelligent and Systems, Bethesda, MD, USA, 1999, pp. 438-443.

[9] Wang Y., Shen D, Teoh E.K. "Lane detection using spline model”, Pattern Recognit. Lett., 2000, Vol. 21, no. 8, pp. 677689.

[10] Yim Y.U., Oh S.Y. "Three-feature based automatic lane detection algorithm (TFALDA) for autonomous driving", IEEE Trans. Intell. Transp. Syst, 2003, Vol. 4 , no. 4,pp. 219225.

[11] Mccall J.C, Trivedi M.M. "Video-based lane estimation and tracking for driver assistance: survey, system, and evaluation", IEEE Trans. Intell. Transp. Syst., 2006, Vol. 7, no. 1, pp. 20-37. 